\title{
Outcome of Arthroscopic Bankart's Repair Using Trans- Glenoid Suture Technique in Recurrent Post-Traumatic Anterior Shoulder Dislocation Without Bony Defect
}

\author{
IA Alabi*, C Nkanta, SA Arojuraye, N Ndubuisi \\ National Orthopedic Hospital, Nigeria \\ *Corresponding author: Ibrahim Abolaji Alabi, Arthroscopy Unit, Department of Clinical Services, National Orthopedic Hospital, Dala, \\ PMB 3087, Dala-Kano, Nigeria.
}

\begin{abstract}
Background: Recurrent dislocation of the shoulder is the leading complication of traumatic anterior glenohumeral dislocation. It occurs commonly in young adult males. The most common pathology encountered in the early phase is Bankart's lesion. Arthroscopic trans glenoid suture capsulorrhaphy is an effective alternative for Correcting the pathology of anterior and anteroinferior shoulder instability.
\end{abstract}

Objective: To present the outcome of arthroscopic Bankart's repair using Caspari's technique.

Methodology: It is a prospective cohort study conducted on 54 patients that undergone arthroscopic Bankart's repair for recurrent shoulder dislocation at National Orthopedic Hospital, Dala and Habeeby Orthopedic Clinic, Kabuga, Kano from March 2015 to February 2021. Cases were recruited through the surgical outpatient department, SOPD. Instability was assessed pre- and postoperatively using the Rowe Score. The instability shoulder index score (ISIS) was used as predictive tool for arthroscopic treatment while the Western Ontario Shoulder Instability Index (WOSI) and the Walch-Duplay scores were used as functional outcome measures. Data were captured using Microsoft excel worksheet and analyzed using SPSS version 20.0 for windows.

Results: Fifty-four patients were studied with an average age of $25.0+/-5.4$ years and M: F of 8: 1 . All were posttraumatic with a mean frequency of dislocation of $5.2+/-3.0$ per week before surgery. All the patients had positive apprehension test. The average duration of hospital stay was $5+/$ - 2 days. The average pre-operative instability shoulder index score (ISIS) was $2.1+/-1.5$. The Rowe scores showed statistical improvement after operation with $\mathrm{P}<0.001$. The average pre-operative Western Ontario Shoulder Instability Index (WOSI) Score and Walch-Duplay scores equally improved to with $\mathrm{P}<0.001$ at 2 years of follow up. There was no recurrence after 2 years of follow up. Caspari's technique is cost effective as two strands of polyester 5 sutures cost $1.4 \%$ of the price of two anchor sutures.

Conclusion: Caspari's Technique gives good to excellent functional outcome in recurrent shoulder dislocations without bony defect and it is cost effective.

\section{Introduction}

Glenohumeral dislocation is a common entity and represents more than $50 \%$ of all joint dislocations with anterior dislocation being most common with overall incidence of $2 \%$ [1]. Dislocation of shoulder involves a tear of the inferior capsuloligamentous complex and labrum from the anterior inferior glenoid most of the times (97\%) [2]. Recurrent instability was defined as at least one episode of recurrent dislocation or subluxation [2]. Recurrent dislocation of shoulder (RDS) is a common injury in high demand professionals, like athletes and military personnel [3]. Recurrent dislocation of the shoulder is the leading complication of anterior glenohumeral dislocation, and it accounts for an average of 70-90\% recurrence rate in patients aged 20 to 40 years [1]. Bankart lesion is found in over $80 \%$ of shoulders with recurrent shoulder instability [1]. The open Bankart repair was the gold standard for years, however arthroscopic Bankart repair has gained popularity [5]. Recurrent instability rates with this type of procedure averaged as low as 7\%. Despite these excellent results, there has been growing interest in the arthroscopic management of anterior glenohumeral instability because of the advantages like less morbidity, shorter time of surgery, improved range of motion, improved cosmesis, 
and less post-operative pain [1]. The main concern in managing traumatic shoulder dislocations is the high rate of recurrence and the functional disability or additional injuries to structures within the glenohumeral joint that may follow [5]. Patient factors including age, activity level, sport and number of instability events must be considered along with examination and imaging findings [6]. The role of arthroscopic procedures in the management of glenohumeral stability continues to evolve [7]. The arthroscopic method offers a less invasive technique of Bankart repair for traumatic anterior shoulder instability [8]. Arthroscopic Bankart repair resulted in a 7-fold lower recurrence rate and a higher rate of return to play than conservative management [9]. Arthroscopic Bankart repair is an effective, cosmetic and safe surgical procedure with good clinical outcomes and excellent post-operative shoulder range of motion [4]. Arthroscopic transglenoid suture capsulorrhaphy, is an effective alternative for correcting the pathology of anterior and anteroinferior shoulder instability [7]. Arthroscopic Bankart's repair in recurrent shoulder dislocation with suture anchors is a reliable procedure with respect to shoulder function, recurrence rate and range of movement [1]. It is best indicated for traumatic anterior dislocators with a Bankart's lesion and good quality glenohumeral ligaments [7]. We study the outcome of Caspari's technique in patients with traumatic recurrent anterior shoulder dislocation without bony defect.

\section{Methodology}

It is a prospective cohort study conducted on 54 patients who underwent arthroscopic Bankart's repair for recurrent shoulder dislocation at National Orthopedic Hospital, Dala and Habeeby Orthopedic Clinic, Kabuga, Kano from March 2015 to February, 2021. All patients aged 18 to 50 years with recurrent posttraumatic anterior shoulder dislocation were recruited through the surgical outpatient department, SOPD. Patients with multidirectional, posterior and fracture dislocation, and those with associated bone defects were excluded from the study. Plain radiographs (true anteroposterior, Rockwood and axillary views, Figure 1ac) were obtained for all the patients to assess bone and articular congruency, rule out bone defects and other degenerative changes. The instability shoulder index score (ISIS) was used as predictive tool for deciding on arthroscopic treatment. Rowe scoring was done to assess instability before surgery as well as WOSI and WalchDuplay scores. The procedure was done under general anesthesia with curved endotracheal tube intubation. patients were positioned in the lateral decubitus position with locally improvised weight over a drip-stand [Figure 2a]. Surgeries were performed by the same surgeon assisted by senior registrars. The standard posterior portal is the portal of entry while the working (anterior) portal is created using an inside-out technique [Figure 2b].
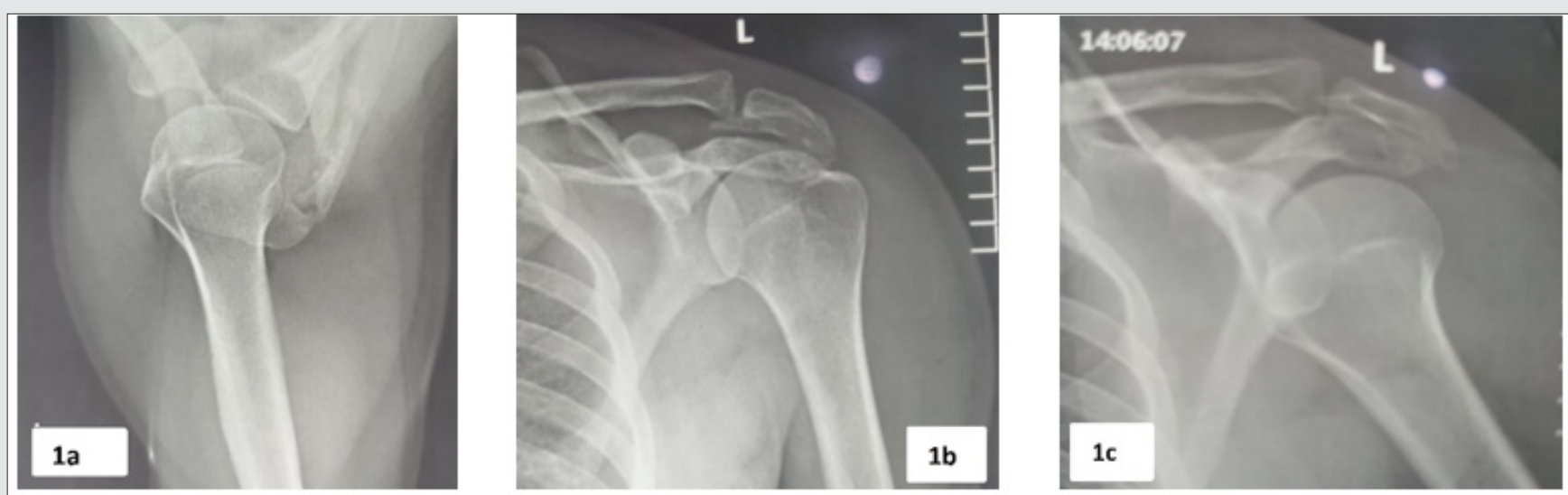

Figure 1 a-c: shows axillary, Anteroposterior and Rockwood views of the left shoulder as part of preoperative assessment in a reduced joint at presentation. Some may present with the dislocated joint.

Figure 2a: shows the anterior view of a patient in the lateral decubitus position after draping; Figure 2b: shows attempt at inside out technique to create the anterior portal. Figure 2c: shows a Bankart lesion. After refreshing the turn surfaces of the labrum and its attachment to the glenoid bone, 2 drilling wires (each with a needle eye) were passed. One is passed through the capsule, through the rotator interval, under the labrum into the glenoid bone, just below the subchondral bone. The other is passed through the same skin incision anteriorly, but at a different inferior point on the capsule, through the subscapularis, under the labrum and into the glenoid bone to the exterior posteriorly. Two strands of polyester 5 sutures are passed parallel and in the same direction through the needle holes [Figure 3a]. The sutures are brought out posteriorly along the holes created by the drills. The sutures are converged to one single hole posteriorly, the glenohumeral joint is deflated and the sutures knotted securely [Figure 3b]. The subcutaneous tissue is released to avoid skin tethering anteriorly and posteriorly. Every patient had a shoulder jack-up brace applied 24 hours after surgery (when swelling had subsided) and maintained on it for 6 weeks [Figure 3c]. Flexion and extension movements were allowed, but not abduction and external rotation until 6 weeks of postoperative period. The shoulder jack-up brace was discontinued at 6 weeks and exercise for full range of motion and muscle strengthening exercises were commenced. The Rowe. WOSI and the Walch-Duplay 
scores were used to assess the shoulder stability and function at 3, 6, 12 and 24 months postoperatively. Patients were granted permission to return to light duties at 3 months and full duty at 6 months. Data were captured using Microsoft excel worksheet and analyzed using SPSS version 20.0 for windows.

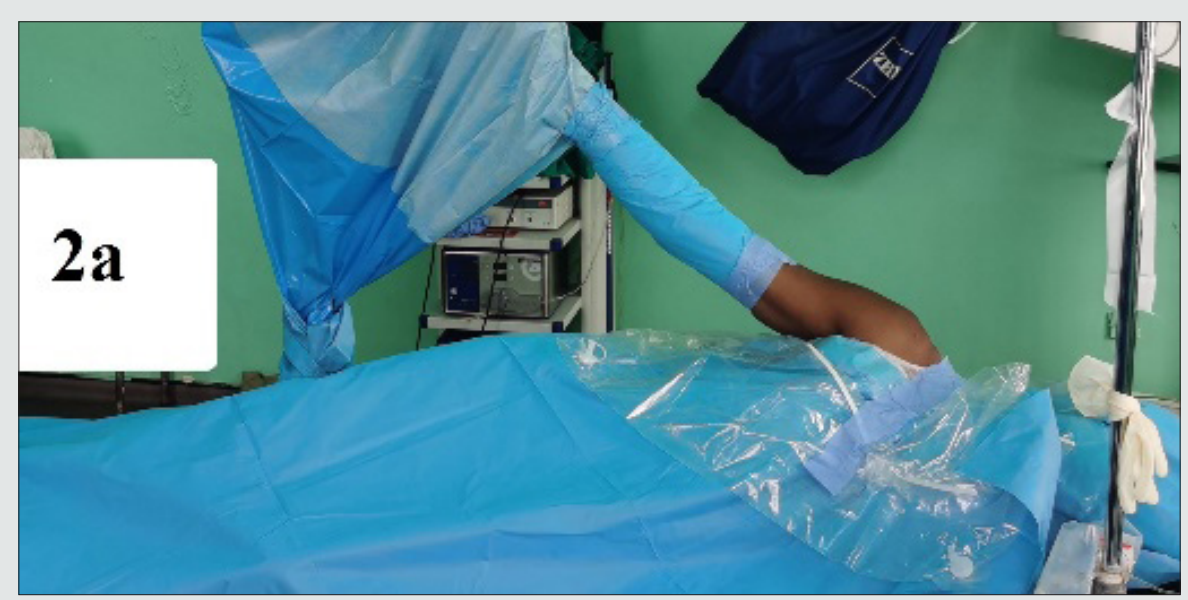

Figure 2a: shows the anterior view of a patient in the lateral decubitus position after draping.

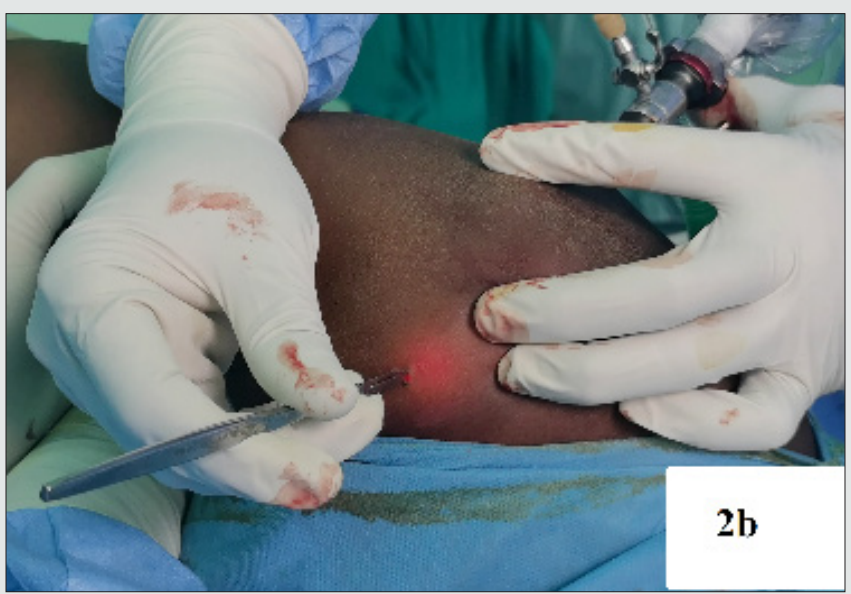

Figure 2b: shows attempt at inside out technique to create the anterior portal.

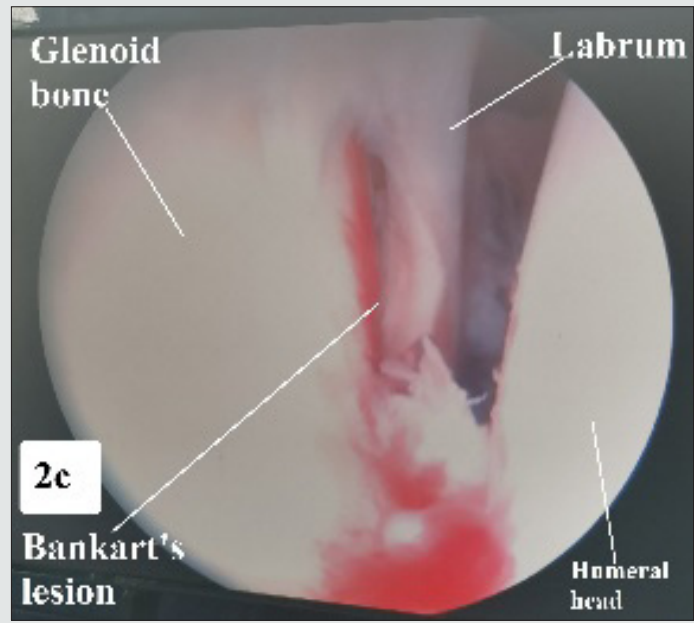

Figure 2c: Shows a Bankart lesion. 


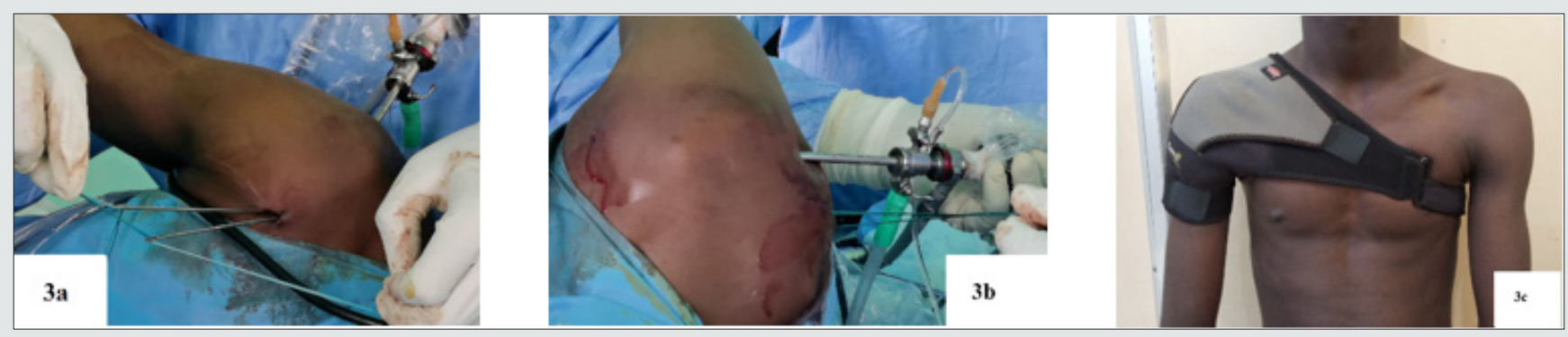

Figure 3a-3c:

\section{Results}

Fifty-four patients were studied with an average age of 25.0 +/- 5.4 years and M: F of 8: 1 [Figure 4]. The right shoulder was 9 times more affected than the left. All were posttraumatic with a mean frequency of dislocation of $5.2+/-3.0$ per week before surgery [Figure 2]. All the patients had had traditional bone setters' manipulation. All the patients had positive apprehension test. The average duration of surgery from skin incision to closure is 55 +/- 15.5 minutes. All the patients were found to have Bankart's lesion with variable degenerative changes. There was significant shoulder swelling extending to the neck region in the immediate postoperative period, but not enough to warrant ICU admission in any of the patients. The swelling subsided in an average of $5.5+$ /-
1.5 hours in all the patients. The average duration of hospital stay was $5+/-2$ days. The average pre-operative instability shoulder index score (ISIS) was $2.1+/-1.5$. The Rowe scores showed statistically significant improvement after operation with $\mathrm{P}<0.001$ at 2 years of follow up [Figure 5]. The average pre-operative WOSI and Walch-Duplay scores were $1444.7+/-271.3(68.8+/-12.9 \%)$ and $27.1+/-9.8$ respectively. The postoperative scores improved to an average of $217.5+/-116.5(10.4+/-5.6 \%)$ and $90.6+/-5.2$ respectively with $\mathrm{P}<0.001$ at 2 years of follow up [Figure 5]. All the patients were followed up for a minimum of 2 years. There was no recurrence of dislocation nor revision after minimum of 2 years of follow up. The cost of 2 strands of polyester 5 sutures needed is 1.4 $\%$ of the cost of 2 anchor sutures.

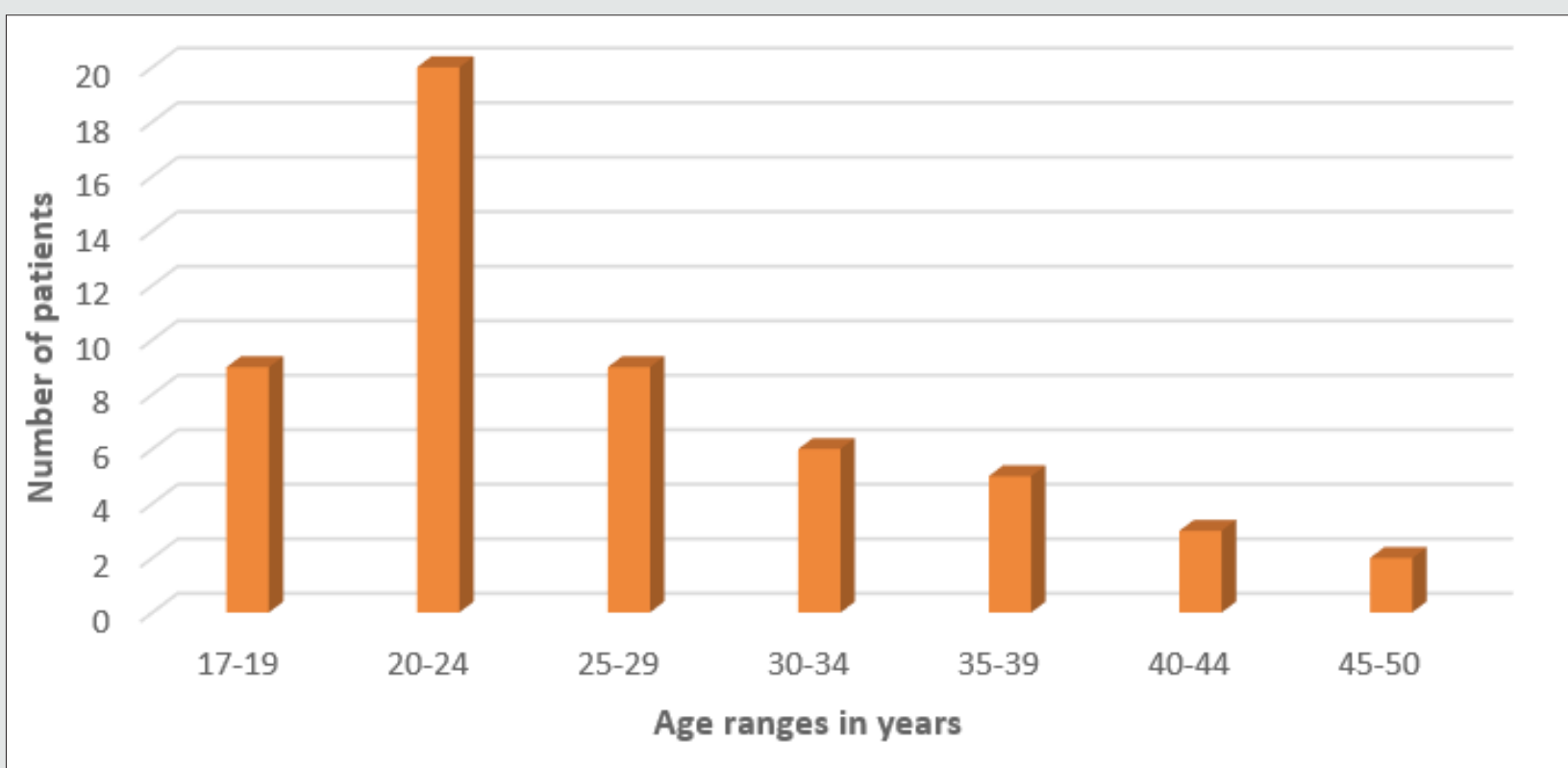

Figure 4: showing the age distribution of the patients studied. 


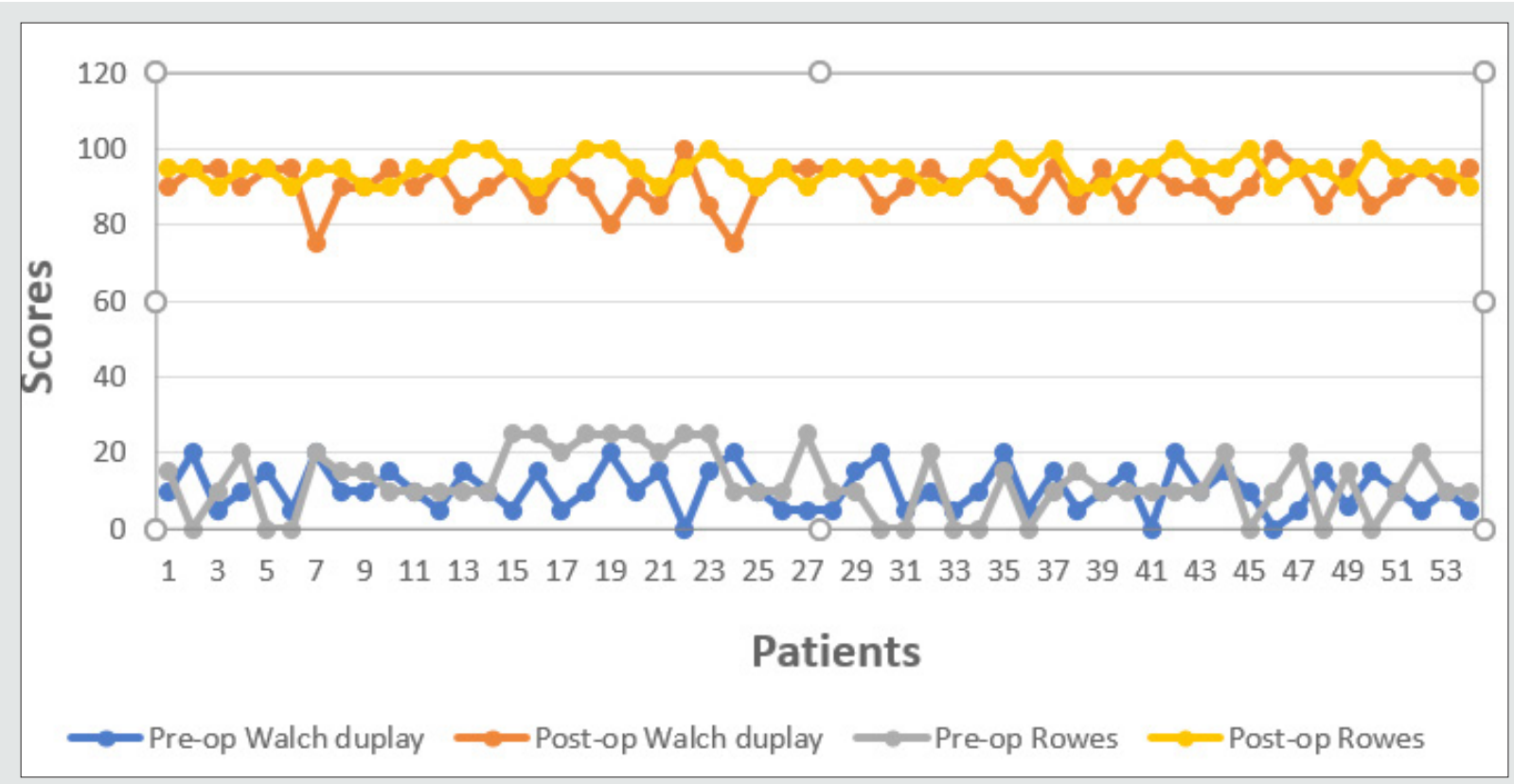

Figure 5: Comparism of Pre- and Postoperative Rowes and Walch-Duplay scores.

\section{Discussion}

Optimal clinical results after arthroscopic Bankart suture repair are mostly dependent on proper patient selection and attention to technical details at the time of surgery [10]. The majority of patients were males (M: F ratio of 8: 1) with an average age of 25.0 +/- 5.4 years which is similar to the findings of Murphy A, et al. where male patients were $75.5 \%$ with average age was 28.0 years (range, 15-73 years) [11] recorded an average age of 21 (16-30) years which suggests more adolescent's involvement than in this study [12]. The average hospital stay was 5 +/- 2days which was more than the 1.2 days (1-5 days) recorded by [12]. This may not be unrelated to the peculiarity of our environments in which there is a wide gap between the tertiary institutions and the primary health care facilities. So patients are kept longer in the tertiary centres than necessary Arthroscopic Bankart repair for anterior shoulder instability has been shown to result in excellent longterm functional outcomes despite a relatively high rate of recurrent instability necessitating revision surgery [11]. Some studies were compared arthroscopic repair to the open Later jet procedure, it was found out that the Later jet procedure had less recurrence [1014]. arthroscopic treatment of patients with recurrent traumatic anterior instability yields results comparable to open procedures, including athletes involved in high-level contact and collision sports. They believed that addressing capsular laxity surgically is critical, particularly when dealing with chronic instability [15]. Our outcome in two years have shown better recurrence rate than the Laterjet procedure at least in the years under review as no recurrence so far. The review by Rossi LA, et al. showed an overall recurrence rate of $11.3 \%$ for arthroscopic Bankart repair based on suture anchors [16]. Arthroscopic Bankart repair with the use of suture anchors is a reliable treatment method, with good clinical outcomes, excellent post-operative shoulder motion and low recurrence rates [8]. Redis location rate tended to be higher if there had been more than 1 dislocation preoperatively ( $\mathrm{P}=.098$ ) [17]. This is however contrary to the findings in this study as mean frequency of dislocation of stood at $5.2+/-3.0$ per week before surgery, but there was no single recurrence at a minimum of 2 years of follow-up. Stabilization after the first-time dislocation achieves better clinical and radiological outcomes than after multiple dislocations [17]. There was no patient who presented with one episode of dislocation. Patients were evaluated before the intervention and at the mean follow-up of approximately two years using Rowe, WOSI and Walch-Duplay scores. Ghai, et al. also used a minimum of 2 years, but used Rowe, Oxford shoulder score and Tegner activity scale [3].

The mean time to return to sports in the study by Rossi LA was 5.3 months [16]. This is similar to return to full duty in 6 months by the patients studied in this study. The demand may not be as enormous as in those involved in collision sports though. The Rowe and ASOSS scores showed statistical improvement after operation $(\mathrm{P}<0.001)$. The ASOSS score varied significantly between sports $(\mathrm{P}<0.001)$ [16]. there is great variability in the rate of return to sport at the same level, in shoulder performance after returning to competition, and in the postoperative recurrence rates [16]. This is similar to our findings where the Rowe scores showed statistical improvement after operation with $\mathrm{P}<0.001$. The average preoperative WOSI and Walch-Duplay scores equally improved to with $\mathrm{P}<0.001$ at 2 years of follow up. Figure 5 showed good correlation between Rowes and Walch-Duplay graphically. All the patients studied were very satisfied with the treatment. This conforms but somewhat better than the findings of Aboalata M, et al. who found out that the patient satisfaction rate was $92.3 \%$ and return to the preinjury sport level was possible in 49.5\% [17-19]. Arthroscopic 
Bankart repair for anterior shoulder instability provides good to excellent functional outcome, improved range of motions and low recurrence rate [8]. Five patients complained of suture-knot irritation in the study by Kim SJ, Jahng JS, Lee JW [9]. Four patients made a similar complain in this study. They were counselled on the temporary nature of such feelings and they all improved clinically. Immediate arthroscopic Bankart repair with an accelerated rehabilitation program is an effective and safe technique for treating young active patients with first-time traumatic anterior shoulder dislocations [10], but it could not be applied here as all the patients presented with more than one episode of dislocation.

\section{Conclusion}

Caspari's Technique gives good to excellent functional outcome in recurrent shoulder dislocations without bony defect and it is cost effective.

\section{References}

1. Kumar DR, Rashid DR, Kumar DK (2021) The clinical and functional outcome of arthroscopic bankart repair in recurrent shoulder dislocation. Int J Orthop Sci 7(1): 147-151.

2. Bessière C, Trojani C, Carles M, Mehta SS, Boileau P (2014) The open Latarjet procedure is more reliable in terms of shoulder stability than arthroscopic Bankart repair. Clinical Orthopaedics and Related Research 472(8): 2345-2351.

3. Ghai A, sachdeva J, Sood M, Sud A, Chauhan M, et al. (2020) Similar functional outcome using single anterior portal and standard two portals technique in recurrent dislocation of shoulder. Chinese J Traumatol 23(2): 102-106.

4. Das A, Bhowmick S, Das N, Samanta S, De S, et al. (2020) Functional outcome of arthroscopic Bankart repair in post-traumatic recurrent shoulder dislocation in a private tertiary care teaching hospital in eastern India. Int J Res Orthop 6(6): 1233.

5. Nelson SY, LeClere LE (2021) Management of the First-Time Shoulder Dislocation. Oper Tech Orthop 30(3).

6. Murray R, Lesniak BP (2020) Arthroscopic Bankart Repair. Oper Tech Orthop 30(3): 100821

7. Rose DJ (1996) Arthroscopic transglenoid suture capsulorrhaphy for anterior shoulder instability. Instructional course lectures 45 p. : 57-64.
8. Ee GWW, Mohamed S, Tan AHC (2011) Long term results of arthroscopic bankart repair for traumatic anterior shoulder instability. J Orthop Surg Res 14(6): 1-28.

9. Hurley ET, Manjunath AK, Bloom DA, Pauzenberger L, Mullett H, et al. (2020) Arthroscopic Bankart Repair Versus Conservative Management for First-Time Traumatic Anterior Shoulder Instability: A Systematic Review and Meta-analysis. Arthrosc - J Arthrosc Relat Surg 36(9): 25262532.

10. Morgan CD (1991) Arthroscopic transglenoid Bankart suture repair. Oper Tech Orthop 1(2): 171-179.

11. Murphy AI, Hurley ET, Hurley DJ, Pauzenberger L, Mullett H (2019) Long-term outcomes of the arthroscopic Bankart repair: a systematic review of studies at 10-year follow-up. Journal of Shoulder and Elbow Surgery 28(11): 2084-2089.

12. Law BKY, Yung PSH, Ho EPY, Chang JJHT, Chan KM, et al. (2008) The surgical outcome of immediate arthroscopic Bankart repair for first time anterior shoulder dislocation in young active patients. Knee Surgery, Sport Traumatol Arthrosc 16(2):188-193.

13. Blonna D, Bellato E, Caranzano F, Assom M, Rossi R, Castoldi F (2016) Arthroscopic Bankart Repair Versus Open Bristow-Latarjet for Shoulder Instability. Am J Sports Med 44(12): 3198-3205.

14. Huxel Bliven KC, Parr GP (2018) Outcomes of the latarjet procedure compared with bankart repair for recurrent traumatic anterior shoulder instability. Journal of Athletic Training 53(2): 181-183.

15. Mishra DK, Fanton GS (2001) Two-year outcome of arthroscopic Bankart repair and electrothermal-assisted capsulorrhaphy for recurrent traumatic anterior shoulder instability. Arthrosc J Arthrosc Relat Surg 17(8): 844-849.

16. Rossi LA, Tanoira I, Gorodischer T, Pasqualini I, Ranalletta M (2020) High Variability in Functional Outcomes and Recurrences Between Contact Sports After Arthroscopic Bankart Repair: A Comparative Study of 351 Patients With a Minimum 3-Year Follow-Up. Arthrosc Sport Med Rehabil 2(5): e575-581.

17. Aboalata M, Plath JE, Seppel G, Juretzko J, Vogt S, et al. (2017) Results of Arthroscopic Bankart Repair for Anterior-Inferior Shoulder Instability at 13-Year Follow-up. Am J Sports Med 45(4): 782-787.

18. Katti DS, Ballal DM, D DHC, A DHK (2017) Functional outcome of arthroscopic Bankart repair for anterior shoulder instability. Int J Orthop Sci 3(1e): 267-271.

19. Kim SJ, Jahng JS, Lee JW (1997) Arthroscopic Transglenoid Bankart Suture Repair with Modifications of Caspari's Technique. Yonsei Med J38(5): 294-300.

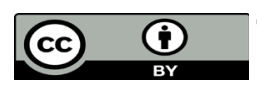

This work is licensed under Creative Commons Attribution 4.0 License

To Submit Your Article Click Here:

Submit Article

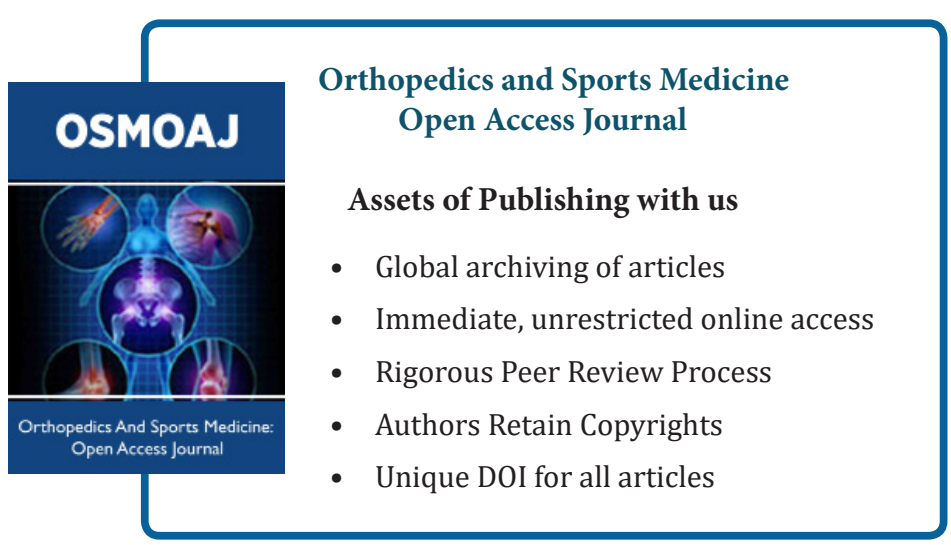

